\title{
A Review of Different Aspects of Covid -19 and Prevention Strategies
}

\author{
Shabnam Akram Sheikh' and Swaroopa Chakole ${ }^{2}$ \\ ${ }^{1}$ Intern, Dept. of Community Medicine, Jawaharlal Nehru Medical College, Datta Meghe Institute \\ of Medical Sciences (Deemed to be University), Sawangi (Meghe), Wardha-442001, Maharashtra, India. \\ 2 Professor, Dept. of Community Medicine, Jawaharlal Nehru Medical College, Datta Meghe Institute \\ of Medical Sciences (Deemed to be University), Sawangi (Meghe), Wardha-442001, Maharashtra, India, \\ Corresponding author email: drswaroopachakole@gmail.com
}

\section{ABSTRACT}

COVID-19 or the coronavirus disease 2019 completed one year since its onset in Wuhan city of the Hubei province of china. Crown shaped virus has proven extremely deadly and has taken millions of lives till date.The test, track, treat approach is followed by government to contain the transmission. But limitations of available beds and deficiencies in existing healthcare infrastructure is hindering the process of handling severe caseloads. Ultimately, prevention proves to be the best option.As the pandemic is progressing, comprehensive study is required to popularize the preventive measures and tell people how they will be effective and benefit them if followed properly. There are various preventive measures which are followed in order to keep the virus at distance. Their effectiveness has been already established in previous outbreaks of SARS, MERS and Ebola. Therefore, this tried and tested methods need to be adopted to take out maximum benefit from them. Already the issue of lack of the required number of health professionals has been lingering from long time. COVID-19 highlighted the need of more health personnel's and maintaining the current strength by protecting them from the viral infection by providing them with suitable equipment's. Long term health implications can be severe in times to come. This article highlights the need of preventive measures to be pursued vigorously so that these unwanted consequences can be averted.

\section{KEY WORDS: PREVENTION, COVID-19, DIETARY GUIDELINES, MEASURES, CARE, HEALTH INFRASTRUCTURE.}

\section{INTRODUCTION}

COVID-19 or the coronavirus disease 2019 completed one year since its onset in Wuhan city of the Hubei province of china. Crown shaped virus has proven extremely deadly and has taken millions of lives till date. The previous outbreaks related to COVID-19 were Severe acute respiratory syndrome (SARS) and Middle Eastern respiratory syndrome (MERS) were only confined to certain geographical location and was only an outbreak.

Biosc Biotech Res Comm P-ISSN: 0974-6455 E-ISSN: 2321-4007

\section{crossef}

Identifiers and Pagination

Year: 2021 Vol: 14 No (6) Special Issue

This is an open access article under Creative

Commons License Attribn 4.0 Intl (CC-BY).

DOI: http://dx.doi.org/10.21786/bbrc/14.6.38
Pages: $174-179$
(Biavardi et al., 2020) But the expanse and its deadly nature of COVID-19 forced World Health Organization (WHO) to declare the outbreak turn epidemic as pandemic in second week of March 2020.

(Covid et al., 2020) As of December 17, 2020 74,289,439 confirmed infection of COVID-19 were reported and $1,650,301$ deaths were reported the crossing one million mark. (Tangcharoensathien et al., 2020) Americas topped the tally only to be followed by Europe and SouthEastAsia. United States of America, India, Brazil, and Russianfederation are the top four placeholder accounting almost half of the infected cases. Huge population density and carelessness and defiance of containment guidelines are the prime factors for such figures to be reported. Non-pharmacological interventions (NPI) were imposed by various agencies in order to break the chain. (Pradhan et al., 2021) These includes lockdown and physical
distancing. After the lifting of restrictions, the infection et al., 2021) These includes lockdown and physical
distancing. After the lifting of restrictions, the infection 
number grew rapidly and caused health care sector to completely collapse.

(Chakraborty et al., 2020) The infection numbers completely overwhelmed the hospitals and clinical facilities that were treating the COVID-19 infection. Therefore, it is important to prevent the infection from happening so all these circumstances can be averted. Huge diagnostics methodologies like test based on swab and blood are employed. Also,several other tests are developed which aids the test, trace, treat model adopted to contain the COVID-19 spread. Diagnostics is first and foremost step in containing the virus. There are various preventive measures which are followed in order to keep the virus at distance. Their effectiveness has been already established in previous outbreaks of SARS, MERS and Ebola. Therefore, this tried and tested method needs to be adopted to take out maximum benefit from them.

Already the issue of less availability of the health professionals has been lingering from long time. COVID19 highlighted the need of more health personnel's and maintaining the current strength by protecting them from the viral infection by providing them with suitable equipment's. Long term health implications can be severe in times to come. Various cardiovascular, pulmonary and neurological symptoms that are persisting for two to three months after getting cured from the virus is serious cause of concern. Therefore, this article highlights the need of preventive measures to be pursued vigorously so that these unwanted consequences can be averted.

Diagnostics Methodologies in Covid-19: Diagnosis is the most important thing in order to start the treatment of the COVID-19. Tremendous spreading capacity and worsening aftereffects of the coronavirus disease 2019 has already made the testing part more important. The test, trace, treat model is solely based on testing outcomes. It should be done as early as possible with randomized testing methodologies in order to screen population or section of population for the COVID-19. If it is not done at early stage then it is not very good for the patients as their health worsens and to health care infrastructure as the spreading ability is multiplied manifold with increase in days before admitting patient to the hospital. Several tests are mentioned in the guidelines rolled out by World Health Organization (WHO) as well as respective country's health department to diagnose people. Reverse transcript polymerase chain reaction (RT-PCR) which works on RNA amplification and if critical threshold (Ct) value is according to guidelines then the concerned patient is said to be harboring the coronavirus.

Nasal or oral swabs are collected by sticks and then put to test. Though chances of manual error and misplacement of samples are there but it is considered as gold standard test in determining whether the person is infected with the coronavirus or not. Rapid antigen antibody test is also employed in diagnosing the population. The blood samples are taken and tested for particular antibodies which are said to be developed after the infection of coronavirus. But it is not the final test as the confirmation of the RT-PCR test is necessary. (Allam et al., 2020) Antibody test is beneficial in checking the outbreak of the diseases in particular hotspot especially in congested urban cluster after which necessary dedicated and targeted efforts can be employed in order to increase the efficiency of the resources used for the containment of the COVID-19.

Other test is also formulated such as test based in saliva, oximeter test but there are ancillary test and need confirmation form aforementioned two tests. To reduce the chances of error, proper training and dissemination of standard operating procedure should be done to health care professionals collecting samples. For example, the load of the virus sometimes shifts to lower end of the respiratory tract then the nasal swab will appear falsely negative. Proper precaution should be taken to avoid these errors. Once the diagnosis is completer and person is tested positive then different treatment courses are followed in symptomatic and asymptomatic patients.

Symptomatic patients which are showing symptoms such as fever, cough, cold and some severe symptoms like loss of taste and smell, diarrhea, vomiting, dyspnea are hospitalized and treated in normal ward in case of former condition and in Intensive care unit if necessary. Isolation and quarantine are two tools available at the disposal of the health authorities which can break the chain of the transmission. Home isolation is also recognized as legitimate way to get people treated due to increasing burden on health care infrastructure. (Guner et al., 2020) Regular visit by trained professionals such as doctors, nurses etc. are made in order to check the patients and see if hospitalization is needed or not.

Preventive Measures: Various methods are undertaken to avoid the infection of the COVID-19. As the outbreak turned pandemic struck suddenly, governing agencies and health authorities tried to curb it by imposing blanket measures like lockdown and physical distancing. (Chu et al., 2020) Then as it was not feasible to continue the lockdown and physical distancing government started to promote other non-pharmacological interventions like wearing of masks, gloves, personal protectiveequipment's (PPE) kits, face shields that permits the people cohabitate with precaution and not getting contracted with the disease. Also, particularly with health professionals and frontline workers these measures are like boon because they are the prime contact with the positive patients. Physical distancing of 6 feet is advised to maintain to ward off the virus transmission.

Six feet apart or six feet under such slogans are promoted to highlight the benefit of the said method. The health professionals and law enforcement agency personnel's need to be provided with advance equipment to keep them secure from the infection. People also need to follow certain guidelines designed by the concerned agencies. Wearing appropriate mask with careful handling is necessary to extract all the benefits of the kit.Sanitization of the surfaces and hands at the regular intervals are necessary in order to avoid any oral transmission. Virus 
can survive on various surfaces starting from two days to few weeks. Vulnerable hotspots like hospitals and public space needs regular sanitization drive. Also, from people sides alcohol-based sanitizers must be handy in order to get their hands sanitized before eating and drinking.

Pregnant women and elderly people are most vulnerable and can develop severe symptoms if necessary precautionary measures are not followed. Infants and new born babies are vulnerable as vertical transmission is not yet established but transmission through mother's sneeze and other human negligence cannot be ruled out. It is widely seen that majority of the infected patients contracted the illnesses by negligence and not following the rules. Also, men developing more critical condition than women are also attributed to the behavioral pattern that are observed by the both. More careless behavior and defiance of rules by male than their female counterpart is one of the reasons of more case fatality rate in men than women.(Gamage et al., 2005) In past outbreak of Ebola in Africa it was only possible to trace the patient and treat them for the disease with the help of personal protective equipment's kits which contain masks and gloves and many other things. Otherwise, nurses and allied health professionals were extremely vulnerable to catch the infected person and get them infected themselves.

Also, the disease is meant to attacking the persons with weaker immune system. (Feyaerts et al., 2020) That means by building immunity and taking appropriate diet and supplement the infection can be ward off by people concern. Foods containing Vitamin C, Vitamin D, (Sundararaman et al., 2020) Probiotics etc. Can be used to build stronger innate immune response system so as it can fight the external invasion of harmful pathogens like coronavirus. Vitamin C is full of antioxidants and antiinflammatory properties which are found in citrus fruits and vegetables in daily diet. (Ali et al., 2020) Vitamin $\mathrm{D}$ not only enables the absorption of the calcium from the food but also aids immune-respiratory functions. Gut bacteria are the essential fauna that our gut harborsand are also essential in absorbing certain elements vital in daily functioning of the body. Various lifestyle changes in pre-COVID-19 and post COVID-19 era has affected due to improper and high sugar and fat containing diet consumption. Essential gut bacteria destroyed by bad habits like consumption of sin goods like alcohol and other psychotropic substances.

Also gut bacteria helps in maintaining the innate immune response in case of external pathogen invasion. Therefore, it is necessary to include supplements like probiotics which rejuvenates the gut health. Probiotics were found to be extremely beneficial in curing the gut disorder. Also, the gut-lung axis which is crucial in COVID-19 and can be decider. Supplementing these through diet or supplement with authentication can be a solution to rising cases. (Butler et al., 2020) Using these as prophylactics can buy some breathing space as the cases are rising exponentially and accommodating all those will be challenge that will be difficult to meet.
Health Care Infrastructure in Covid-19: In the test, trace, treat model, the last bit is the important one in containing and curing the positive patients. The fact that it is one of the unprecedenteddiseases spread in past couple of centuries is due to its immensely fast spreading capacity and deadly nature. In fact, the case fatalities are among the highest in past century. Although the case fatality rate is quite low comparably but the widespread nature and number of infected cases also are huge making the number hit a million mark unfortunately. Initially the outbreak in Wuhan city was not seen properly but when epicenter changed from China to European countries particular in Italy and Spain, the world was terrified as the casualties hit the mark of ten thousand in a single day.

This was due to the fact that the health care infrastructure was overwhelmed with the patient's load that it was not able to handle such number of patients. Considerable number of patients was not even treated as due to unavoidable circumstances they could not get the bed. Virtually the health care infrastructure was completely collapsed. In densely populated countries like India, initially the imposition of preventivemeasures like lockdown and movement restriction bought some time for governing agencies to think on the containment strategy and boost the health care infrastructure. As the lockdown started to ease the case numbers shot up exponentially, creating need of additional beds that could accommodate all the patients. Particularly in metro cities like Mumbai where congestion of population or population density is huge and physical distancing like measures were ineffective and clusters of the outbreak were created, difficult to handle at the same time.

So, the need of preventing the disease from happening at first place was felt. Wearing mask and gloves with proper physical distancing were popularize in order to underline and convey the benefits of the same. Severe acute respiratory syndrome (SARS-COV-2)affects the respiratory system of the body. Therefore, the need oxygen support system and ventilators were there in case of severely ill patients. As the comorbidity which is largely prevalent, is the deciding factor in deciding the severity of the illness. Therefore, the demand for the sophisticated medical equipment's were high whereas the availability was and is quite low. (Blumenthal et al., 2020) Prevention is the only feasible and affordable option available right now till the vaccine arrives to deal with such grim scenario.

Health care professionals and front-line workers like policing agency personnel are highly vulnerable to the infection. It is necessary to protect them to avoid the infection. Already various reports have highlighted that even in developed countries the health care personnel per unit population is low leave the case of least developed countries where this number stands at very low level. Therefore, it is necessary to take care especially by these above-mentioned professionals to protect them as well as maintain the treatingprofessional's number. (Blumenthal et al., 2020) According to international council of 
nurses, the casualties occurred during COVID-19 duty of nurses has already exceeded the number of nurses died during Second World War. The loss of health care professionals affects the containment plan manifold as we lose our frontline workers which are already less in number. Therefore, prevention yet again is always better than cure.

Long Term Implications Of Covid-19: A year has passed since the outbreak of the disease in Wuhan city of china, still not much is known about the disease. From changing symptoms through fever, cough and cold and diarrhea, dyspnea, the uncertainty attached with the disease is quite high. Only few solid correlationshave been drawn till date and many more needs to be establish after thorough study. Vaccine procedure are in their last phases and some are ready for approval but it will take time as distribution and logistics are huge challenges ahead. Further mass production of the same would be a difficult and time-consuming task. Even if people are vaccinated there is no solid information about the disease's long term health implications after testing positive for the disease. Certain reports from Europe have registered some severe symptoms and uneasiness after the people got treated from the disease.

Erratic and disturbed sleep cycle, losing and getting back taste and smell indicators, fatigue are the changes repotted by the people who got treated for the disease. (Del Rio et al., 2020) After testing repeatedly they tested negative. Other reportedly registered symptoms were found through various tests done under study. Ringing sensation in the ear, intermittent brain freeze, not able work at stretch more than few hours, palpitations, mood swings were reported by considerable number of people even after recovering from the COVID-19 and that too up tofifty-sixty days after recovering from the disease. (Yelin et al., 2020) A study found out that 78 out of 100 patients were showing abnormal findings in their cardiovascular MRI after getting cured from COVID-19. Unusually prolonged fatigue was common feature among the recovered patients along with reduced physical capacity. Al though the source of these complaints is still unanswered and are largely being related to after effects of COVID-19.Extensive need of post COVID-19 rehabilitative care may shoot up in coming days.

Pulmonary after effects of the COVID-19 is also a cause of concern. Ina study of over 55 patients 60 days after discharge, a quarter of patients were showing decrease carbon monoxide diffusion capacity with diminishing respiratory muscle strength. Neurologic implications occur due to direct diffusion through olfactory indicator or indirectly through blood vessels. Chemosensory dysfunction, seizures, encephalitis are some of the symptoms that showed up after two to three months after discharge. SARS-COV-1, MERS also had neurological symptoms lingering months after recovering from the respective disease. (Gopalini et al., 2021, Agrawal et al., 2020, Bagde et al., 2020, Bakshi et al., 2020, Motwani et al., 2021) A number of reviews reported the different aspects of prevention in terms of Covid-19. (Mishra et al., 2021) Balwani et. al.

reported on assessment of fear psychosis and practice modification in dental fraternity to fight against COVID-19.(Bhokardankar et al., 2020, Bhutada et al., 2020, Nisargandha et al., 2020, Deogada et al.,2020) Many studies emphasized the importance of immunity boosting through different ways. An emotional aspect is also attached with the onset of the COVID-19 infection. (Johnson et al., 2020) The isolation and quarantine measures left the patient lonely till the treatment to stop the transmission of the disease can take serious toll on the mental health of the concerned patients. (Nagvi et al., 2020, Kute et al., 2020, Khubchandani et al., 2020, Kasturkar et al., 2020, Jachak et al., 2020, Hande et al., 2020) Many reviews were reported regarding Covid-19 and it's health related aspects. Especially in case of pregnant women where two lives are at stake and to be mother is already worried about the baby, COVID-19 infection only adds to the woes of the pregnant mother. So various aspects are at stake and it is advisable to take proper precaution to avoid the infection.

\section{CONCLUSION}

COVID-19 pandemic has reached every nook and corner of the world affecting each and every individual in direct or the indirect way. Initially the challenge was to contain the viral spread and treat the people who got infected somehow. But recent trends are worrying. Overwhelming health infrastructure was also coupled by long term health implications of COVID-19. Persistence of various symptoms even after getting cured for 2-3 months needs extensive study to find out the reason behind the same. Still the pandemic is creating new trends to study and is quite uncertain for prediction. Preventive measures can be the solution to these problems. Complacency is gaining root day by day as the time is passing. People are being careless and not following various containment guidelines which are necessary to be followed such as wearing masks, sanitizing hands regularly, maintain physical distancing and so on. These persisting symptoms needs to be popularized so that people are aware of these and follow the guidelines.

Because long term health implications will require additional attention by medical fraternity and also the individual will also suffer mentally and physically. Vaccine is still a distant dream for majority of the people so preventive measures need not be dropped off. Infect inculcating hygienic habits and maintain the healthy dietary intake can protect people from not only this pandemic but also in future such scenario as WHO has already stated that COVID-19 is not the last pandemic that humanity will face. Wearing mask and sanitizing hands regularly is found to be helpful in other communicable diseases and can protect the individual from falling ill with particular preventable diseases saving mental as well as monetary costs that would be incurred by the individual otherwise. To sum 
up prevention is always better than cure especially in disease outbreaks like COVID-19 which has attached huge medical, social, economic implications are attached. Further forming hygienic habits will also serve as a tool to fight any kind outbreaks that may be prevented by following best practices.

\section{REFERENCE}

Agrawal, D., Jaiswal, P. and Bajaj, P., 2020. Role of dentist to stop COVID-19: A journey map. International Journal of Research in Pharmaceutical Sciences, 11(Special Issue 1).

Ali, N., 2020. Role of vitamin D in preventing of COVID-19 infection, progression and severity. Journal of infection and public health.

Allam, M., Cai, S., Ganesh, S., Venkatesan, M., Doodhwala, S., Song, Z., Hu, T., Kumar, A., Heit, J. and Coskun, A.F., 2020. COVID-19 diagnostics, tools, and prevention. Diagnostics, 10(6), p.409.

Bagde, K., Rathi, B., Rathi, R., Badwaik, P. and Khabde, S., 2020. Role of common kitchen remedies in prevention of infectious diseases with a particular perspective of COVID-19. International Journal of Research in Pharmaceutical Sciences, 11(Special Issue 1).

Bakshi, S., Toshniwal, V., Agrawal, A., Acharya, S. and Shukla, S., 2020. Awareness and Psychosocial Effects of COVID-19 Pandemic on Health Care Professionals and Medical Students Across the State of Maharashtra. Int J Cur Res Rev| Vol, 12(22), p.122.

Bhokardankar, P., Rathi, B., Khan, M. and Rathi, R., 2020. COVID-19 pandemic: Home remedies for immunity boosting. International Journal of Research in Pharmaceutical Sciences, 11(Special Issue 1).

Bhutada, R.S., Rathi, R. and Dasar, D., 2020. Immunity boosting diet during Covid 19. International Journal of Research in Pharmaceutical Sciences, 11(Special Issue 1).

Biavardi, N.G., 2020. Being an Italian medical student during the Covid-19 outbreak. International Journal of Medical Students, 8(1), pp.49-50.

Blumenthal, D., Fowler, E.J., Abrams, M. and Collins, S.R., 2020. Covid-19-implications for the health care system.

Blumenthal, D., Fowler, E.J., Abrams, M. and Collins, S.R., 2020. Covid-19-implications for the health care system.

Butler, M.J. and Barrientos, R.M., 2020. The impact of nutrition on COVID-19 susceptibility and long-term consequences. Brain, behavior, and immunity.

Chakraborty, P.S., Ravi, S. and COVID, S., 2020. 19| Is India's health infrastructure equipped to handle an epidemic.

Chu, D.K., Akl, E.A., Duda, S., Solo, K., Yaacoub, S.,
Schünemann, H.J., El-harakeh, A., Bognanni, A., Lotfi, T., Loeb, M. and Hajizadeh, A., 2020. Physical distancing, face masks, and eye protection to prevent person-to-person transmission of SARS-CoV-2 and COVID-19: a systematic review and meta-analysis. The Lancet, 395(10242), pp.1973-1987.

COVID, C., 2020. Global Cases [Internet]. Johns Hopkins Center for Systems Science and Engineering.

Del Rio, C., Collins, L.F. and Malani, P., 2020. Longterm health consequences of COVID-19. Jama, 324(17), pp.1723-1724.

Deogade, M.S., 2020. INTERNATIONAL JOURNAL OF RESEARCH IN PHARMACEUTICAL SCIENCES.

Feyaerts, A.F. and Luyten, W., 2020. Vitamin C as prophylaxis and adjunctive medical treatment for COVID-19?. Nutrition, 79, p.110948.

Gamage, B., Moore, D., Copes, R., Yassi, A., Bryce, E. and BC Interdisciplinary Respiratory Protection Study Group, 2005. Protecting health care workers from SARS and other respiratory pathogens: a review of the infection control literature. American journal of infection control, 33(2), pp.114-121.

Gopalani, S., 2021. Covid-19-Impact across Nations and Sectors. Annals of the Romanian Society for Cell Biology, pp.6986-6994.

GÜNER, H.R., Hasano囚lu, I. and Akta囚, F., 2020. COVID19: Prevention and control measures in community. Turkish Journal of medical sciences, 50(SI-1), pp.571577.

Hande, A., Sonone, A., Gadbail, A., Gawande, M., Patil, S. and Sharma, P., 2020. Modalities to restrain the progression of oral potentially malignant diseases and oral squamous cell carcinoma in COVID-19 pandemic. Oral Oncology.

Jachak, S.P., Phansopkar, P.A., Naqvi, W.M. and Kumar, K., 2020. Great Awakening--Telerehabilitation in Physiotherapy during Pandemic and Impact of COVID19. Journal of Evolution of Medical and Dental Sciences, 9(45), pp.3387-3394.

Johnson, G. and Tabner, A., 2020. Mitigating the Psychological Impact of COVID-19 on Healthcare Workers: A Digital Learning Package.

KASTURKAR, P.R. and GAWAI, J.P., 2020. Engaging School Going Children During COVID-19 Lockdown. Journal of Clinical \& Diagnostic Research, 14(8).

Khubchandani, S.R. and Dahane, T.M., 2020. Emerging Therapeutic Options for COVID-19. Journal of Evolution of Medical and Dental Sciences, 9(41), pp.3082-3086. Kute, V., Guleria, S., Prakash, J., Shroff, S., Prasad, N., Agarwal, S.K., Varughese, S., Gupta, S., Gokhale, A.G.K., Sahay, M. and Sharma, A., 2020. NOTTO transplant specific guidelines with reference to COVID-19. Indian Journal of Nephrology, 30(4), p.215. 
Latchoumi, T.P., Ezhilarasi, T.P. and Balamurugan, K., 2019. Bio-inspired weighed quantum particle swarm optimization and smooth support vector machine ensembles for identification of abnormalities in medical data. SN Applied Sciences, 1(10), pp.1-10.

Mishra, P., 2021. Fake News-A Pandemic within a Pandemic. Psychology and Education Journal, 58(2), pp.629-636.

Motwani, A. and Chakole, S., 2021. COVID-19Community Based Mitigation Measures. Psychology and Education Journal, 58(2), pp.478-486.

Naqvi, W.M. and Sahu, A., 2020. Paradigmatic Shift in the Education System in a Time of COVID 19. Journal of Evolution of Medical and Dental Sciences, 9(27), pp.1974-1977.

Nisargandha, M.A. and DadaraoParwe, S., 2020. Spread of coronavirus disease 2019 (COVID-19) during thelockdownintheIndianpopulationandpreventive measures. International Journal of Research in
Pharmaceutical Sciences, 11, pp.328-332.

Pradhan, S. and Chakole, S., 2021. Preventive Measures for Health Professionals in COVID-19. Psychology and Education Journal, 58(2), pp.757-764.

Sundararaman, A., Ray, M., Ravindra, P.V. and Halami, P.M., 2020. Role of probiotics to combat viral infections with emphasis on COVID-19. Applied microbiology and biotechnology, pp.1-16.

Tangcharoensathien, V., Calleja, N., Nguyen, T., Purnat, T., D’Agostino, M., Garcia-Saiso, S., Landry, M., Rashidian, A., Hamilton, C., AbdAllah, A. and Ghiga, I., 2020. Framework for managing the COVID19 infodemic: methods and results of an online, crowdsourced WHO technical consultation. Journal of medical Internet research, 22(6), p.e19659.

Yelin, D., Wirtheim, E., Vetter, P., Kalil, A.C., Bruchfeld, J., Runold, M., Guaraldi, G., Mussini, C., Gudiol, C., Pujol, M. and Bandera, A., 2020. Long-term consequences of COVID-19: research needs. The Lancet Infectious Diseases, 20(10), pp.1115-1117. 